\title{
Estudio exploratorio del impacto de la red social en el nivel individual de aceptación de sistemas empresariales
}

\author{
Exploratory study of the impact of the social network at the individual \\ level of acceptance of enterprise systems \\ Patricio Ramírez Correa ${ }^{1} \quad$ Jorge Alfaro Pérez ${ }^{2}$ \\ Recibido 3 de enero de 2012, aceptado 2 de abril de 2012 \\ Received: January 3, 2012 Accepted: April 2, 2012
}

\begin{abstract}
RESUMEN
El presente trabajo tiene por propósito explorar el impacto de la red social en el uso de sistemas empresariales. Utilizando un modelo de aceptación de tecnologías de información con base en TAM, se explora la red de relaciones sociales asociadas al uso efectivo del sistema empresarial. El modelo de investigación incorporó las siguientes variables: normas sociales, ansiedad computacional, demostrabilidad de los resultados, relevancia para el trabajo, percepción de utilidad, percepción de facilidad de uso, intención de uso y uso del sistema. En una muestra de 90 individuos se realizó un análisis SEM-PLS del modelo que incluyó los valores asociados a medidas de centralidad y densidad de la red. El modelo de investigación y los resultados iniciales de este análisis se exponen en este artículo.
\end{abstract}

Palabras clave: TAM, análisis de redes sociales, sistemas empresariales, aceptación individual, SEM-PLS.

\section{ABSTRACT}

The purpose of this paper is to explore the impact of the social network in using enterprise systems. Using a model of information technology acceptance based on TAM, this paper explores the social network associated with the effective use of the enterprise system. The research model incorporated the following variables: social norms, computer anxiety, results demonstrability, job relevance, perceived usefulness, perceived ease of use, behavior intention to use and use of the system. In a sample of 90 individuals, the SEM-PLS analysis of the model included the values associated with measures of centrality and network density. The research model and initial results of this analysis are presented in this article.

Keywords: TAM, social networks analysis, enterprise systems, individual acceptance, SEM-PLS.

\section{INTRODUCCIÓN}

Las tecnologías de información (TI) otorgan a las organizaciones capacidades que en conjunto con la interacción de las habilidades del usuario pueden apoyar a crear valor sustentable. Los sistemas empresariales son un tipo particular y destacado de TI para la empresa, y si bien se les reconoce mundialmente un gran impacto en los resultados de la organización, este impacto depende de su aceptación a nivel individual.

El estudio académico de este fenómeno es reciente pero es de gran relevancia para la práctica. Por ejemplo, en el caso particular de una empresa que desee aproximarse al resultado de un proyecto de implantación de un nuevo sistema empresarial es fundamental explorar el nivel de aceptación

1 Escuela de Ingeniería Comercial. Universidad Católica del Norte. Larrondo 1281, Coquimbo, Chile. E-mail: patricio.ramirez@ucn.cl

2 Departamento de Ingeniería de Sistemas y Computación. Universidad Católica del Norte. Av. Angamos 0610, Antofagasta, Chile.E-mal: jalfaro@ucn.cl 
individual de sus actuales sistemas empresariales. El diagnóstico de esta realidad entregará elementos claves a considerar, tanto en la selección de software y consultores como en la implantación y operación del nuevo sistema.

Propuesto por Fred Davis [1], el modelo de aceptación de la tecnología (TAM) explica el proceso de aceptación de la tecnología de información a nivel individual. TAM postula que la intención de conducta individual de uso de la tecnología de información queda determinada por la utilidad percibida y la facilidad de uso percibida. Al mismo tiempo, la facilidad de uso percibida afecta directamente la utilidad percibida.

Diversos autores han estudiado la aceptación de TI bajo el modelo TAM en el ambiente de sistemas empresariales, y fruto de estos estudios se han descrito antecedentes específicos que influyen en el mayor uso de tales sistemas en la empresa. Adicionalmente, se han desarrollado aproximaciones que incorporan variables asociadas a la red social de los usuarios de estos sistemas como antecedente de su uso [2].

El presente trabajo tiene por propósito explorar el impacto de la red social en el uso de sistemas empresariales. En particular, el estudio analizará la relación entre centralidad y densidad de la red de apoyo en el uso de un sistema empresarial en una empresa de servicios. El artículo se ha estructurado en la siguiente forma. Primero, se presenta formalmente el modelo TAM. A continuación se describe el modelo de investigación, incluyendo cada una de sus variables. Seguidamente se describe el trabajo de campo, para finalizar con los resultados y conclusiones del estudio.

\section{MODELO TAM}

En el contexto científico, se entiende un modelo como una representación simplificada de una realidad elaborada a partir de conceptos previamente definidos y cuyo propósito último es proporcionar los términos, relaciones y proposiciones de tal realidad. Es una "red" que lanzamos para apresar, racionalizar y explicar el mundo que nos rodea. TAM (Technology Acceptance Model-Modelo de Aceptación de la Tecnología) es un modelo que intenta explicar por qué los individuos utilizan (o no utilizan) las tecnologías de información que tienen a su disposición [1]. Desarrollado a partir elementos de la sicología, el modelo TAM ha sido utilizado en las últimas dos décadas para explicar con éxito este fenómeno de aceptación [3].

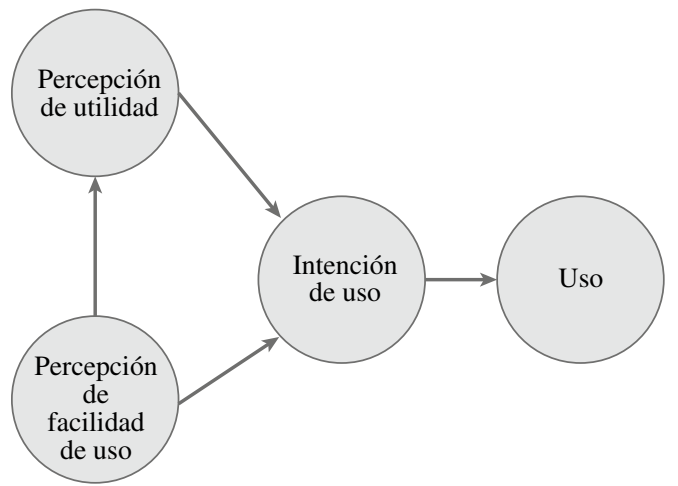

Figura 1. Modelo TAM

La Figura 1 muestra el modelo TAM en su forma básica. Este modelo se puede explicar como sigue. $\mathrm{Al}$ aumentar la percepción de facilidad de uso aumenta la percepción de utilidad de las TI, y al aumentar estas dos percepciones aumenta la intención de uso. Un aumento de la intención de uso está directamente relacionado con el uso real de la TI.

\section{RED SOCIAL Y USO DE UN SISTEMA}

Los resultados de [2] indican que la inserción de un empleado en la red social de una empresa que implementa un nuevo sistema de información puede mejorar la comprensión del uso de tal tecnología.

Según estos autores, los compañeros de trabajo de un individuo pueden ser una fuente importante de ayuda para superar barreras de conocimiento que restringen el uso de un sistema complejo. Por tanto, la densidad de la red (que refleja relaciones de obtener ayuda de un empleado), y la centralidad de la red (que refleja relaciones de dar ayuda de un empleado) son antecedentes clave del uso del sistema.

\section{DESCRIPCIÓN DEL MODELO DE INVESTIGACIÓN E HIPÓTESIS}

La Figura 2 muestra el modelo de investigación utilizado en este estudio. El modelo está basado en TAM (en verde) e incorpora a él un conjunto de variables antecedentes (en celeste). 


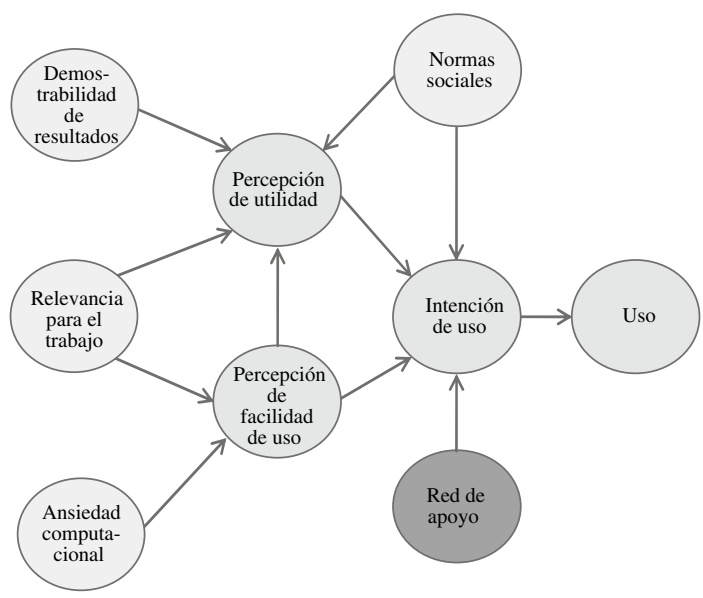

Figura 2: Modelo de investigación

En el modelo un aumento en la demostrabilidad de los resultados y en la relevancia para el trabajo del sistema aumenta la percepción de utilidad. Además, la percepción de facilidad de uso aumenta si aumenta la relevancia para el trabajo del sistema, como también aumenta si disminuye la ansiedad computacional. Finalmente, un aumento de la variable normas sociales incide positivamente en la percepción de utilidad y la intención de uso del sistema. Por otra parte, se ha incorporado un antecedente asociado a la red de apoyo en el uso del sistema (en naranja), un aumento en las características de densidad y centralidad de esta red implicarían un aumento en la intención de uso.

Basados en [2, 3], a continuación se definen las variables del modelo:

1. Percepción de facilidad de uso (PEOU): Grado de facilidad asociada con el uso del sistema.

2. Percepción de utilidad (PU): Grado en que una persona cree que usar el sistema le ayuda a obtener ganancias en el desempeño laboral.

3. Uso (USE): Nivel de utilización de sistema.

4. Intención de uso (BI): Grado en que una persona ha formulado planes conscientes de usar o no usar en el futuro un sistema.

5. Ansiedad computacional (CANX): Grado de aprensión de un individuo, o incluso el miedo, cuando él o ella se enfrentan a la posibilidad de utilizar un computador.
6. Normas sociales (SN): Percepción del individuo asociada a que la mayoría de las personas que son importantes para él creen que debe (o no debe) usar el sistema.

7. Relevancia para el trabajo (JREL): Percepción individual en relación con el grado en que el sistema es relevante para su trabajo.

8. Demostrabilidad de resultados (RES): Tangibilidad de los resultados de utilizar un sistema.

9. Red de apoyo (NET): Centralidad y densidad de la red de apoyo para utilizar un sistema.

Basados en [1, 2, 3], las hipótesis de este estudio son las siguientes:

- H1: Un aumento de la intención de uso aumenta el uso real de los sistemas empresariales.

- H2: Un aumento de la percepción de facilidad de uso aumenta la intención de uso de los sistemas empresariales.

- H3: Un aumento de la percepción de utilidad aumenta la intención de uso de los sistemas empresariales.

- H4: Un aumento de la percepción de facilidad de uso aumenta la percepción de utilidad de los sistemas empresariales.

- H5: Un aumento en la demostrabilidad de los resultados aumenta la percepción de utilidad de los sistemas empresariales.

- H6: Un aumento en la relevancia para el trabajo del sistema aumenta la percepción de utilidad de los sistemas empresariales.

- H7: Un aumento en la relevancia para el trabajo del sistema aumenta la percepción de facilidad de uso de los sistemas empresariales.

- H8: Un aumento de la ansiedad computacional aumenta la percepción de facilidad de uso de los sistemas empresariales.

- H9: Un aumento de normas sociales aumenta la percepción de utilidad de los sistemas empresariales. 
- H10: Un aumento de normas sociales aumenta la intención de uso de los sistemas empresariales.

- H11: Un aumento en los valores de centralidad y densidad de la red de apoyo aumenta la intención de uso de los sistemas empresariales.

\section{DESCRIPCIÓN DEL TRABAJO DE CAMPO}

\section{Muestra}

El trabajo de campo se realizó en el mes de enero de 2011 a través de una encuesta en línea a los usuarios del sistema empresarial de la empresa X. La empresa $\mathrm{X}$, importante organización de servicios de carácter privado y con sede en la ciudad de Antofagasta (Chile), tiene aproximadamente 300 empleados y su sistema empresarial está en operación hace más de cinco años.

Se envió la solicitud de respuesta a cada uno de los usuarios de la empresa y como resultado del proceso se obtuvieron un total de 73 encuestas válidas. Para efectos del análisis de redes sociales, y dada la estructura de la encuesta, estos 73 encuestados referenciaron a 90 funcionarios de la empresa.

\section{Escalas de medida}

Las escalas de medición aplicadas para medir los constructos o variables latentes del modelo han sido probadas en otras investigaciones [2,3]. Salvo para la variable RED, todas las escalas utilizadas fueron tipo Likert (siete puntos). A continuación se detallan los ítems de cada escala.

Percepción de facilidad de uso (PEOU)

- PEOU1 La interacción con el sistema empresarial es clara y comprensible.

- $\quad$ PEOU2 Aprender a operar el sistema empresarial es fácil.

- PEOU3 El sistema empresarial es fácil de usar.

- PEOU4 Es fácil llegar a ser diestro en el uso del sistema empresarial.

Percepción de utilidad (PU)

- PU1 El sistema empresarial permite realizar mis tareas más rápidamente.

- PU2 El sistema empresarial mejora mi desempeño laboral.
- PU3 El sistema empresarial incrementa mi productividad.

- PU4 El sistema empresarial mejora mi efectividad laboral.

- PU5 El sistema empresarial hace más fácil hacer mi trabajo.

- PU6 El sistema empresarial es útil en mi trabajo.

Uso (USE)

- USE1 En promedio, ¿cuánto tiempo pasa en el sistema empresarial cada día?

- USE2 En promedio, ¿cuántas veces al día ocupa el sistema empresarial?

Intención de uso (BI)

- BI1 Suponiendo que tenga acceso al sistema empresarial, tengo la intención de usarlo.

- BI2 Suponiendo que tenga acceso al sistema empresarial, puedo predecir que voy a utilizarlo.

- BI3 Voy a utilizar el sistema empresarial en los próximos meses.

Ansiedad computacional (CANX)

- CANX1 Trabajar con un computador me pone nervioso.

- CANX2 Los computadores me hacen sentir inquieto.

- CANX3 Los computadores me hacen sentir incómodo.

Normas sociales (SN)

- SN1 Mi supervisor inmediato piensa que yo debería utilizar el sistema empresarial en mi trabajo.

- $\quad$ SN2 Las gerencias y jefaturas han sido de ayuda en el uso del sistema empresarial.

- SN3 En general, la organización ha apoyado el uso del sistema empresarial.

Relevancia para el trabajo (JREL)

- JREL1 En mi trabajo, el uso del sistema empresarial es importante. 
- JREL2 En mi trabajo, el uso del sistema empresarial es relevante.

- JREL3 El uso del sistema empresarial es pertinente a las diversas tareas relacionadas con mi trabajo.

Demostrabilidad de resultados (RES)

- RES1 No tengo ninguna dificultad para decir a otros acerca de los resultados de utilizar el sistema empresarial.

- RES2 Yo creo que podría comunicar a otros los resultados de utilizar el sistema empresarial.

- RES3 Los resultados de utilizar el sistema son claros y evidentes para mí.

En este estudio la variable Red de Apoyo (NET) se operacionaliza en cuatro medidas de la red de usuarios: centralidad (NC), centralidad valorada (VNC), densidad (ND) y densidad valorada (VND).

\section{RESULTADOS DE LA EVALUACIÓN DE LA ACEPTACIÓN DE TI}

\section{Estadísticas descriptivas por ítems}

La Tabla 1 muestra las estadísticas de uso de los encuestados.

Tabla 1. Uso de sistemas empresariales en la empresa

\begin{tabular}{|c|c|c|}
\hline \multicolumn{3}{|c|}{ Nivel de Uso Percibido $(\mathbf{N}=73)$} \\
\hline Alto & Medio & Bajo \\
\hline 36 & 22 & 15 \\
\hline
\end{tabular}

La Tabla 2 muestra la media y desviación estándar de los ítems de las variables del modelo de investigación.

\section{Estadísticas por variable}

La Tabla 3 muestra estadísticas para las variables del modelo.

Desde una perspectiva metodológica, la Tabla 3 indica a través de los índices AVE, Confiabilidad Compuesta, Alfa de Cronbachs y Comunidad que los ítems consultados explican en forma razonable a la variable medida. Desde una perspectiva práctica, el valor del índice de la Tabla 3 resume el nivel de las variables en estudio.
Tabla 2. Ítems (de 1 a 7)

\begin{tabular}{|c|c|c|}
\hline Е & $\frac{. \pi}{\tilde{E}}$ & $\begin{array}{l}\dot{\Xi} \\
\text { 苞 }\end{array}$ \\
\hline PU1 & 4,59 & 1,698 \\
\hline PU2 & 4,90 & 1,529 \\
\hline PU3 & 4,66 & 1,643 \\
\hline PU4 & 4,52 & 1,642 \\
\hline PU5 & 4,59 & 1,580 \\
\hline PU6 & 5,26 & 1,405 \\
\hline PEOU1 & 5,00 & 1,236 \\
\hline PEOU2 & 5,32 & 1,235 \\
\hline PEOU3 & 5,25 & 1,278 \\
\hline PEOU4 & 5,34 & 1,145 \\
\hline USE1 & 4,30 & 1,713 \\
\hline USE2 & 4,60 & 2,146 \\
\hline CANX1 & 1,52 & 1,056 \\
\hline CANX2 & 1,52 & 1,107 \\
\hline CANX3 & 1,47 & 1,055 \\
\hline BI1 & 6,03 & ,971 \\
\hline BI2 & 5,85 & 1,232 \\
\hline $\mathrm{BI} 3$ & 6,23 & 1,007 \\
\hline SN1 & 6,10 & 1,030 \\
\hline SN2 & 4,63 & 1,541 \\
\hline SN3 & 4,88 & 1,666 \\
\hline JREL1 & 6,15 & 1,050 \\
\hline JREL2 & 6,03 & 1,154 \\
\hline JREL3 & 5,97 & 1,080 \\
\hline RES1 & 5,89 & 1,074 \\
\hline RES2 & 5,88 & 1,027 \\
\hline RES3 & 5,58 & 1,246 \\
\hline
\end{tabular}

\section{Análisis SEM-PLS}

Con el propósito de valorar el modelo de investigación, el primer análisis empírico realizado confirmó la razonable fiabilidad de las escalas (Alfa de Cronbachs > 0.7).

En consideración del propósito y restricciones de nuestro estudio seleccionamos la técnica PLS (Partial Least Square) para contrastar las hipótesis de la investigación $[4,5,6]$. El software SmartPLS 2.0 de M3 se utilizó para este análisis [7]. 
Tabla 3. Variables (Índice de 1 a 7)

\begin{tabular}{|c|c|c|c|c|c|}
\hline 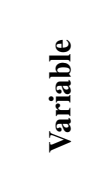 & 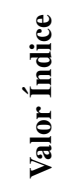 & $\sum$ & 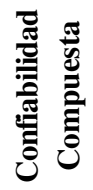 & 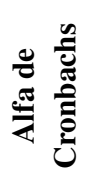 & 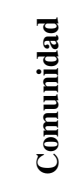 \\
\hline BI & 6,05 & 0,75 & 0,90 & 0,83 & 0,75 \\
\hline CANX & 1,50 & 0,98 & 0,99 & 0,99 & 0,98 \\
\hline JREL & 6,05 & 0,91 & 0,97 & 0,95 & 0,91 \\
\hline PEOU & 5,23 & 0,78 & 0,93 & 0,91 & 0,78 \\
\hline PU & 4,76 & 0,85 & 0,97 & 0,97 & 0,85 \\
\hline RES & 5,79 & 0,80 & 0,92 & 0,89 & 0,80 \\
\hline SN & 5,29 & 0,66 & 0,85 & 0,74 & 0,66 \\
\hline USE & 4,44 & 0,92 & 0,96 & 0,91 & 0,92 \\
\hline
\end{tabular}

La primera fase fue la valoración del modelo de medida, en ella confirmamos tanto fiabilidad individual de cada ítem y constructo, como validez convergente y validez discriminante de todos los constructos. En una segunda fase procedimos a evaluar si el modelo estructural apoya al modelo de investigación propuesto.

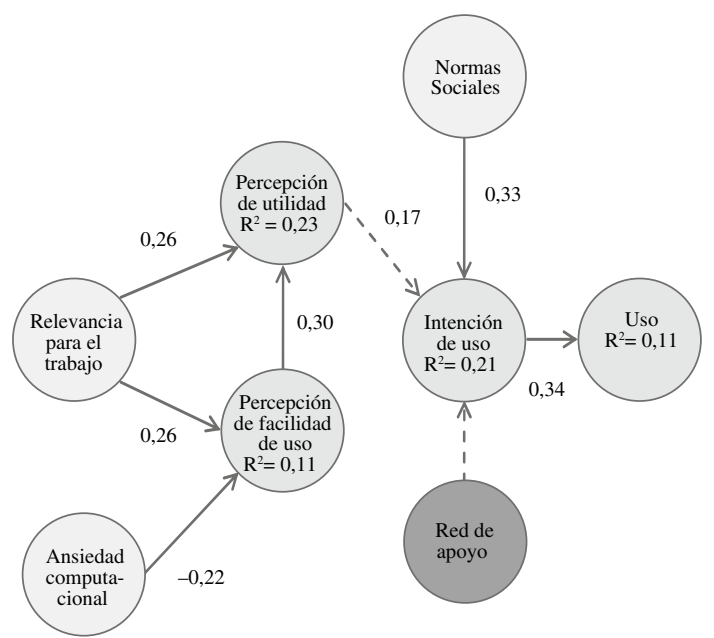

Figura 3. Modelo valorado.

La Figura 3 muestra los caminos estructurales con valores significativos y el valor de los $\mathrm{R}^{2}$. La Tabla 4 muestra el resultado del proceso de bootstrap para el cálculo de la fiabilidad de los caminos estructurales.

Tal como muestra la Tabla 4, los resultados confirman mayoritariamente las relaciones que se establecieron en el modelo de investigación.
Tabla 4. Coeficientes de los caminos (paths).

\begin{tabular}{|c|c|c|c|}
\hline 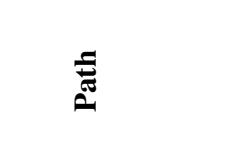 & $\stackrel{\pi}{0}$ & 异 & $\dot{0}$ \\
\hline BI -> USE & 0,34 & 7,73 & $* * *$ \\
\hline CANX -> PEOU & $-0,22$ & 3,11 & $* *$ \\
\hline JREL -> PEOU & 0,26 & 6,16 & $* * *$ \\
\hline JREL -> PU & 0,26 & 4,77 & $* * *$ \\
\hline $\mathrm{NC}->\mathrm{BI}$ & 0,28 & 4,25 & $* * *$ \\
\hline $\mathrm{ND}->\mathrm{BI}$ & $-0,33$ & 4,35 & $* * *$ \\
\hline PEOU -> BI & 0,02 & 0,44 & n.s. \\
\hline PEOU -> PU & 0,30 & 6,46 & $* * *$ \\
\hline $\mathrm{PU}->\mathrm{BI}$ & 0,17 & 2,38 & $*$ \\
\hline RES -> PU & 0,06 & 1,09 & n.s. \\
\hline $\mathrm{SN}->\mathrm{BI}$ & 0,33 & 5,95 & $* * *$ \\
\hline $\mathrm{SN}->\mathrm{PU}$ & 0,02 & 0,29 & n.s. \\
\hline $\mathrm{VNC} \rightarrow \mathrm{BI}$ & 0,07 & 2,02 & $*$ \\
\hline VND $->$ BI & 0,35 & 6,10 & $* * *$ \\
\hline
\end{tabular}

\section{Análisis de redes sociales}

Para el contraste de H11 se analizó la red de usuarios de la empresa en función de su nivel de apoyo al uso del sistema. Utilizando los valores asociados a medidas de centralidad (NC y VNC) y densidad (ND y VND) de la red se examinó el modelo de investigación del estudio. Acorde con [2], estos valores se determinaron en base a consultar a cada encuestado la lista de personas a las que solicitaba ayuda y la lista de personas a las que daba ayuda para usar el sistema empresarial, y luego pedirles que valoraran a cada uno de los integrantes de estas listas según el nivel de conocimiento, información y recursos para operar eficientemente el sistema empresarial de la empresa.

Como resultado del cálculo de medidas de redes sociales, tal como se ve en la Figura 3 y Tabla 4, se determinó que en general las asociaciones en esta red de apoyo aumentan las intenciones de uso del sistema.

En particular, el grado de centralidad en la red (NC) y el grado de densidad valorado (VND) de cada entrevistado se revelan como antecedentes positivos de la intención de uso del sistema. Sin embargo, el grado de densidad (ND) de cada entrevistado se revela como antecedente inverso de la intención de uso del sistema. 
La Tabla 5 resume el contraste de hipótesis.

Tabla 5. Contraste de hipótesis.

\begin{tabular}{|c|c|}
\hline Hipótesis & Contraste \\
\hline $\begin{array}{l}\text { H1: Un aumento de la intención de } \\
\text { uso aumenta el uso real de los sistemas } \\
\text { empresariales. }\end{array}$ & Soportada \\
\hline $\begin{array}{l}\text { H2: Un aumento de la percepción de } \\
\text { facilidad de uso aumenta la intención } \\
\text { de uso de los sistemas empresariales. }\end{array}$ & No soportada \\
\hline $\begin{array}{l}\text { H3: Un aumento de la percepción de } \\
\text { utilidad aumenta la intención de uso } \\
\text { de los sistemas empresariales. }\end{array}$ & $\begin{array}{c}\text { Débilmente } \\
\text { soportada }\end{array}$ \\
\hline $\begin{array}{l}\text { H4: Un aumento de la percepción } \\
\text { de facilidad de uso aumenta la } \\
\text { percepción de utilidad de los sistemas } \\
\text { empresariales. }\end{array}$ & Soportada \\
\hline $\begin{array}{l}\text { H5: Un aumento en la demostrabilidad } \\
\text { de los resultados aumenta la } \\
\text { percepción de utilidad de los sistemas } \\
\text { empresariales. }\end{array}$ & No soportada \\
\hline $\begin{array}{l}\text { H6: Un aumento en la relevancia } \\
\text { para el trabajo del sistema aumenta la } \\
\text { percepción de utilidad de los sistemas } \\
\text { empresariales. }\end{array}$ & Soportada \\
\hline $\begin{array}{l}\text { H7: Un aumento en la relevancia } \\
\text { para el trabajo del sistema aumenta } \\
\text { la percepción de facilidad de uso de } \\
\text { los sistemas empresariales. }\end{array}$ & Soportada \\
\hline $\begin{array}{l}\text { H8: Un aumento de la ansiedad } \\
\text { computacional aumenta la percepción } \\
\text { de facilidad de uso de los sistemas } \\
\text { empresariales. }\end{array}$ & Soportada \\
\hline $\begin{array}{l}\text { H9: Un aumento de normas sociales } \\
\text { aumenta la percepción de utilidad de } \\
\text { los sistemas empresariales. }\end{array}$ & No soportada \\
\hline $\begin{array}{l}\text { H10: Un aumento de normas sociales } \\
\text { aumenta la intención de uso de los } \\
\text { sistemas empresariales. }\end{array}$ & Soportada \\
\hline $\begin{array}{l}\text { H11: Un aumento en los valores de } \\
\text { centralidad y densidad de la red de } \\
\text { apoyo aumenta la intención de uso de } \\
\text { los sistemas empresariales. }\end{array}$ & $\begin{array}{c}\text { Soportada } \\
\text { Parcialmente }\end{array}$ \\
\hline
\end{tabular}

\section{CONCLUSIONES}

De este estudio se pueden extraer las siguientes conclusiones iniciales.

Desde una perspectiva práctica, los usuarios de la empresa $X$ tienen una alta intención de uso de sus sistemas, los mismos que evalúan positivamente en relación a la relevancia de éstos en su trabajo; por otra parte, estos usuarios tienen una baja ansiedad al momento de enfrentar los computadores y perciben medianamente útiles y fácil de usar los sistemas actuales.

Desde una perspectiva académica, se ha podido apoyar al modelo TAM como buen representante de la aceptación de los sistemas empresariales. Diversos elementos pueden ser extraídos del análisis de este modelo, por ejemplo, un aumento en la relevancia para el trabajo del sistema aumenta indirectamente el uso del sistema.

Adicionalmente, el análisis de redes sociales aplicado indica qué mecanismos de copia y de influencia social se presentan como importantes para aumentar la intención de uso del sistema empresarial en la empresa, y si bien los resultados de estos análisis son preliminares, dan elementos de juicio cuantitativos para futuros trabajos al respecto.

\section{REFERENCIAS}

[1] F.D. Davis. "Perceived usefulness, perceived ease of use, and user acceptance of information technologies". MIS Quarterly. Vol. 13, Issue 3, pp. 319-340. 1989.

[2] T.A. Sykes, V. Venkatesh and S. Gosain. "Model of acceptance with peer support: A social network perspective to understand employees'system use". MIS Quarterly. Vol. 33, Issue 2, pp. 371-393. 2009.

[3] V. Venkatesh and H. Bala. "Technology Acceptance Model 3 and a Research Agenda on Interventions". Decision Sciences. Vol. 39, Issue 2, pp. 273-315. 2008.

[4] W.W. Chin. "The partial least squares approach for structural equation modeling". En George A. Marcoulides (Ed.), Modern Methods for Business Research, Lawrence Erlbaum Associates. 1998. 
[5] M. Tenenhaus, V.E. Vinzi, Y-M. Chatelin and C. Lauro. "PLS path modeling". Computational Statistics and Data Analysis. Vol. 48, pp. 159-205. 2005.

[6] C. Fornell and D.F. Larcker. "Evaluating structural equation models with unobservable variables and measurement error". Journal of Marketing Research. Vol. 18, pp. 39-50. 1981.

[7] C.M. Ringle, S. Wende and A. Will. "SmartPLS 2.0 (M3) beta". Hamburg. 2005. URL: http://www.smartpls.de 